\title{
Intelligent Design and Metrology for Higher Quality, Accuracy and Improving Production Efficiency
}

\author{
M. Numan Durakbasa (A) durakbasa@mail.fft.tuwien.ac.at \\ P. Herbert Osanna (A)
}

BIOGRAPHICAL NOTES

M. Numan Durakbasa, prof. Dr. Dipl. -Ing. Prof.h.c. (born in 1955) Head of the Department for Interchangeable Manufacturing and Industrial Metrology (Austauschbau und Messtechnik / Produktionsmesstechnik \& Qualität) of the Institute for Production Engineering and Laser Technology, and Nanotechnology Laboratory and vice head of the Department for Certification of Quality Professional at Vienna University of Technology, with long-standing international practical experience as "Quality System Auditor", Austrian expert in the international technical Committee ISO/TC 213 "Geometrical Product Specifications and Verification", as well as ISO/TC 176 "Quality Management and Quality Assurance", chairman of the Austrian Standard Committee ON-K 031 "Anforderungen und Prüfungen der geometrischen Produktspezifikation; Technische Produktdokumentation" and expert of the Austrian Standard Committees ON-K 129"Quality Management Systems" and "Environmetal Management Systems". Research Interests: metrology, quality management, geometrical product specifications and verification - GPS, nanometrology, CAQ, environmental management, interchangeable manufacturing, industrial metrology, measurement technique, precision engineering, calibration, accreditation, certification, standardisation. Publications: 9 books, more than 280 scientific papers.

\section{KEY WORDS}

Intelligent metrology, Design, GPS, Accuracy, Quality, Production Efficiency

\section{ABSTRACT}

Adequate knowledge in the areas of intelligent metrology and design are important presuppositions to achieve waste free production and low costs of manufacturing and accuracy at the same time within the sophisticated production systems. This is of extreme importance in present time of worldwide international competition in industry and production engineering and at the same time increasingly higher costs of energy and raw material. The prescription and consumption of material and energy to achieve the necessary and required workpiece accuracy in series manufacturing depends to a great extent from the (geometrical) workpiece tolerances of any kind (roughness, form, positional, dimensional) which are prescribed for the production and the fulfillment of these tolerances and therefore for the function of the produced workpieces and their fitness for practical application and none the less of the economy of production altogether. This requirement is of great importance at the time being which is characterized as described above.

\section{Accuracy, Efficiency and High Quality in Production}

The problematic of the high accuracy of the work pieces in modern industrial production technique gained in the last years more and more importance through constantly 
increasing demands on the quality of the produced parts. The necessity faces to the additional outlay caused through that in the entire manufacturing process, to produce due to the world wide competition fight's and it price pressure's resultant from that maximally economical and to strive for cost savings and efficiency increasing in production. If the workpiece geometry of machined parts is considered as a whole there exist interactions between the different features forming the periphery of the part. But also within the surface of every single feature there exist interactions between geometrical deviations of different kind and different order. If we take these deviations of dimensions, roughness, form and position collectively the existing interactions are significant for the accuracy, high quality and the functions of the parts that should be accomplished during practical application. The most important parameters in determining the suitability of a technical part are its compatibility, functionality, performance and corrosion resistance. The precise assessment of wear, friction and miniaturization demands creation of nanometer scaled surface structures, surfaces with thin film deposition and ultra precision surface treatment with the utilization of new manufacturing and measurement instrumentation and techniques. These include micro and nanofabrication of surface patterns and topographies by the use of laser machining, photolithographic techniques, and electron beam and colloidal lithography to produce controlled structures on technical surfaces in size ranging from $10 \mathrm{~nm}$ to $100 \mu \mathrm{m}$. At the time being 3D surface measurement is already proved to be an important tool in several areas of surface analysis including wear, indentation, topography, contact problems and functional behavior of surfaces (see Fig. 1).

The needs of the industry for ultra-high precision engineering and workpieces with a surface roughness less than few nanometers call for measurement instrumentation that can be applied reliably in modern production processes, together with international standards defining parameters and tolerances in the nanometer scale. The requirements on the measurement systems and the measurement strategy to determine suitable parameters, time, costing and the guarantee of a predetermined process stability by means of measurable and correlated parameters come into focus.

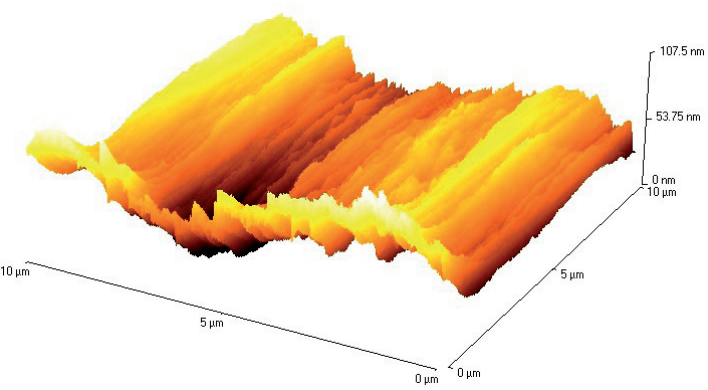

Fig. 1 3D Measurement of a high precision machined alloy specimen

\section{Production Engineering and Production Me- trology - from Past to Future}

Since 1970 we see increasing importance of modern metrology as means to control and improve industrial manufacturing and the quality of all kinds of products and processes to test technical products with high accuracy on the basis of geometrical product specifications and verification. At the same time precision engineering developed as important trend in instrumentation and metrology.

As the tolerances of workpieces and their features decrease, the interaction and correlation between dimensional tolerances and surface finish becomes more important [1]. To achieve surface finishes and part tolerances in the sub-micrometer and nanometer level, it is necessary to incorporate very sophisticated instrumentation and metrology into the design [2].

In the same period the standards governing product design and manufacturing have undergone basic international harmonisation. Focal points of interest included workpiece microgeometry [3] and geometrical deviations [4], as well as tolerancing principles according to the principle of independence [5]. In many countries, the above-mentioned international standards have been adopted also on a national level, similar as the new international standards about quality management $[6,7,8]$.

In this respect the general term "Geometrical Product Specifications and Verification GPS" has become recently well-known for the area of mechanical engineering. It defines on a technical drawing the shape (geometry), dimensions and surface characteristics of the workpiece under discussion. In this way the optimal function of the respective part is supposed to be guaranteed considering certain manufacturing tolerances. Nevertheless workpieces will be produced, which do not fulfill these require- 
ments. Therefore workpieces are measured in order to compare them with the specifications. There is a need to relate between actual workpieces and: nthe workpiece imagined by the designer, the workpiece as manufactured, the knowledge about the workpiece as measured. In order to establish this relationship between design, production and measurement and to clarify the mutual importance, standards have been developed in the area of Geometrical Product Specifications and Verification. Comprehensive knowledge in this area is an important presupposition to achieve economic design, construction, production, metrology and quality management (see Fig. 2).

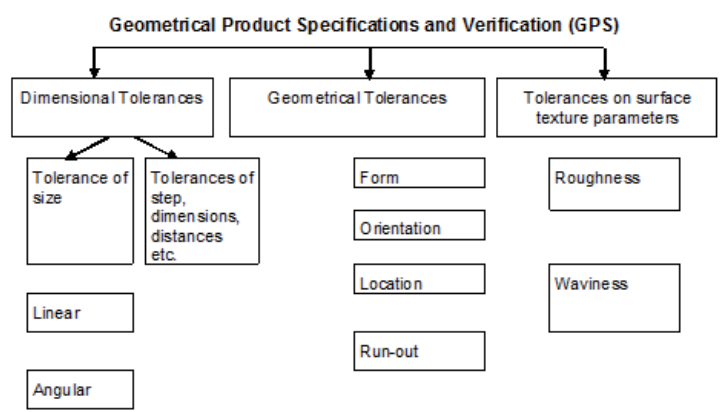

Fig. 2 Geometrical Tolerances and Tolerances of Dimension and Geometrical Properties of the Surface

As already mentioned above this set of requirements concerning the geometry of the workpiece (or of an assembly of several workpieces) is known as "GPS" covering requirements of size and dimension, geometrical tolerances and geometrical properties of the surface.

A feasible approach to new international measurement standards for roughness and toleration on the nanometer scale would be the adaptation and transfer of the GPS (Geometrical Product Specifications and Verification) to nano metrology. The GPS Technical Committee of ISO was established in June 1996 with the aim to create a "Masterplan" which summarizes all existing geometric standards [9]. In this masterplan there are the 18 most important geometric parameters listed, each of them with the appropriate standards. Similar to this approach in conventional geometric metrology, a masterplan for the most important geometric parameters in nano metrology could be designed and then serve as the basis for the definition of new international measurement standards in this area.

The concept of GPS is represented in Fig. 3, showing four different types of GPS standards and designated as the "GPS-matrix-model" (see Fig. 2) [9].

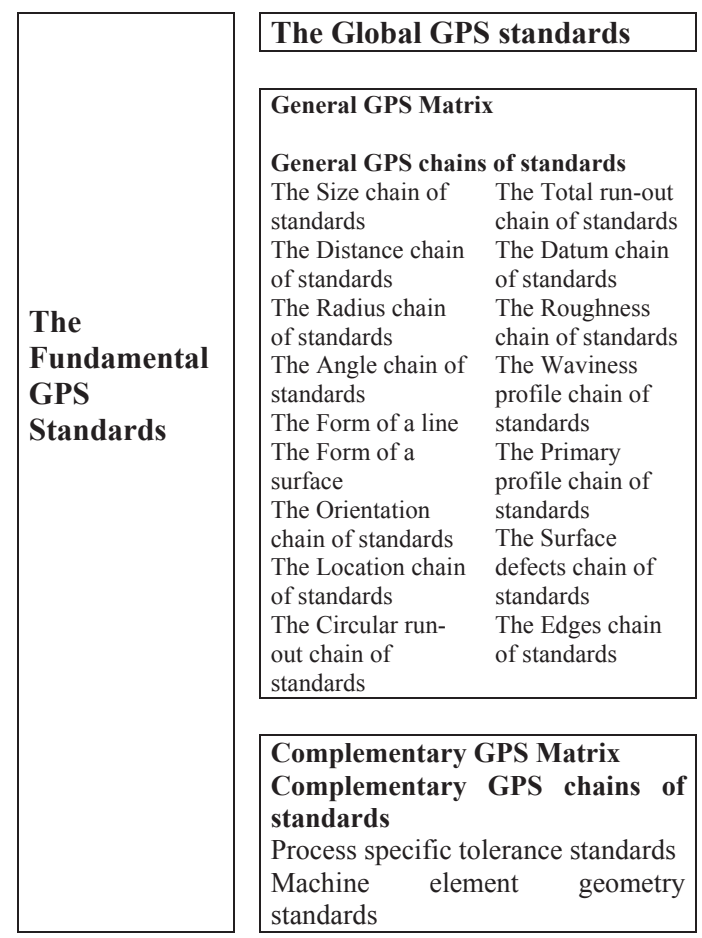

Fig. 3 The GPS-matrix-model - GPS Masterplan - Overview

\section{Geometrical Product Specifications are a means to trans- form function dependent demands into produced work- pieces and parts based on: \\ - mathematical rules and methods, \\ - consideration of macro and micro geometry, \\ - possibilities for measuring of quantities and espe- cially toleranced quantities and \\ - evaluation of uncertainty, etc.}

\section{Co-ordinate Metrology in Modern Production Environment}

An important development as far as workpiece metrology is concerned is the big general advance of coordinate metrology which also happened in the same period of time as computer-aided metrology and "GPS" in general.

Three dimensional coordinate measuring machines (3D-CMMs) allow to measure deviations of dimensions, form and position very accurately with only one measuring device $[10,11]$. For 3D measure- 
ments it is often more advantageous to use a CMM Such Nano-CMMs have been recently introduced achieving an uncertainty of $100 \mathrm{~nm}$ or better and enabling three-dimensional measurements as well as scanning of high precision parts.

Besides measuring accuracy the number of workpieces to be measured is important when choosing the measuring device. Especially when workpiece tolerances are more accurate than tolerance grade IT5 (e.g. $11 \mu \mathrm{m}$ for $50 \mathrm{~mm}$ it is necessary to make use of coordinate metrology. This is also possible for big series of workpieces.

CMMs are referred to as those measuring instruments giving physical representations of a three dimensional rectilinear Cartesian coordinate system.

\section{The nature of coordinate metrology can be defined as:}

The geometrical features of the workpiece to be measured are touched in several measuring points using a coordinate measuring device.

The coordinates of the measuring points are used to compute the mathematical geometry of the workpiece with help of the control computer of the CMM. At the time being coordinate metrology is a very important tool to solve problems of production metrology of nearly any kind especially when high flexibility and high accuracy are demanded at same type of workpiece. One of the essential requirements in coordinate metrology is the computation of associated features from the probed contact points.

When we consider as an example the measurement of a bore hole, we can distinguish between conventional measurements and the application of coordinate metrology. A two-point-measurement has to be done by means of the strategy trying to get the maximum value perpendicular to the axis and then finding the minimum in the axial section (see Fig. 4). The bore diameter is given by the condition that these two diameter values are equal.
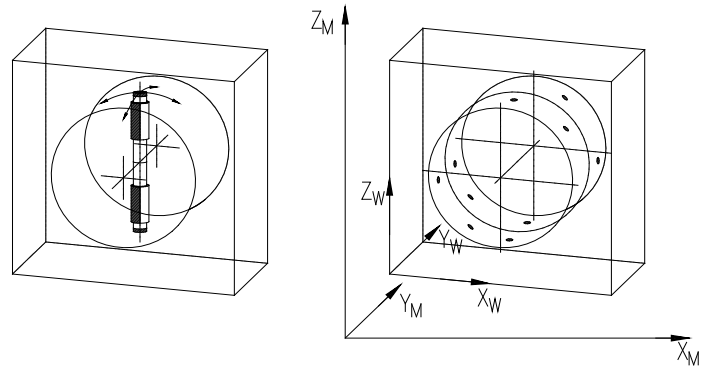

Fig. 4 Conventional Metrology and Coordinate Metrology

\section{In this context it is distinguished between:}

nominal geometry,

real geometry and

n substitute geometry.

This follows the way of parts from design to manufacturing and quality control (see Fig. 5).

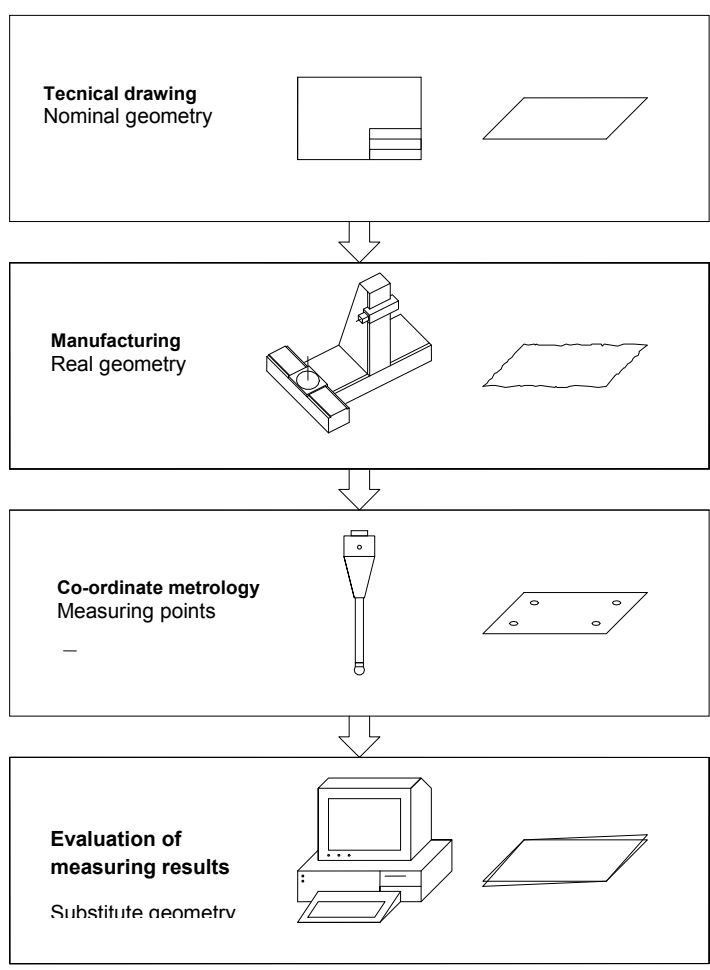

Fig. 5 Principle of the Workpiece evaluation in the Frame of Geometrical Product Specifications and Verification

\section{Intelligent Metrology with Coordinate Mea- suring Machines}

The course of the steps, which are necessary for a measurement, is always identical independent of the kind of the programming. Choosing the stylus combination is an essential step for the measurement. It must occur on the one hand so that all geometrical features to be measured can be touched, on the other hand too complicated and particularly too long stylus combinations are to be avoided since an additional source of errors arises from their bending.

The steps which are needed for the automated measurement of workpieces are shown in Fig. 6.

The measurement results obtained with several or even many different probes is then as that obtained with a single probe tip with the diameter zero. By 
using an automatic stylus-exchanging-unit every combination needs to be calibrated only once if there are not any extremely high demands on the uncertainty of measurement.

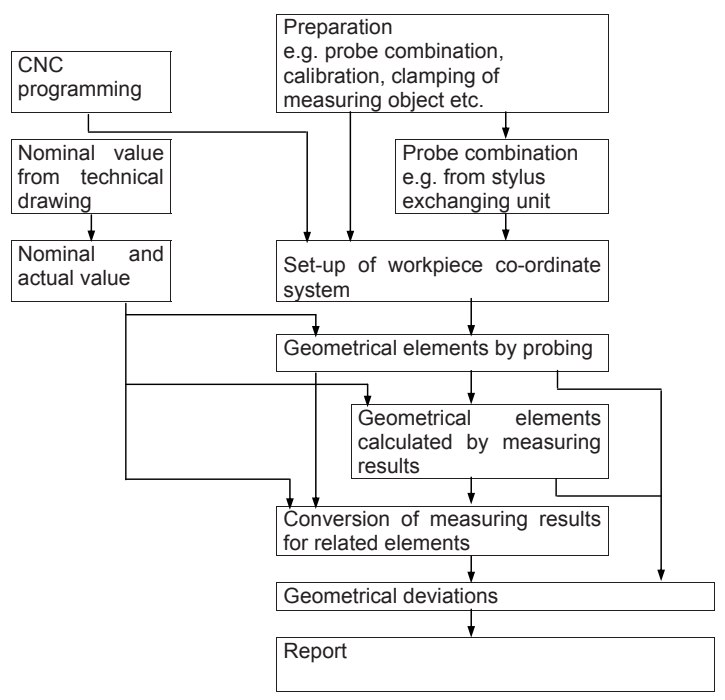

Fig. 6 Flow Diagram for the Measurement Steps on CMMs

\section{Outlook to Future Developments}

The ideas presented in this publication explain in principal the correlation between different geometrical deviations and the manufacturing conditions. This can help to achieve lower manufacturing costs and at the same time higher quality, accuracy and efficiency in present production.

The results of the presented study can be seen as a further step in the direction of a comprehensive analysis of workpiece geometry and it is fully in line with research work already carried out in the past [11]. By the described successful application of co-ordinate metrology for the solution of measurement problems of various kinds also new challenges are put onto precision production measurement technology especially in the area of GPS.

\section{References}

[1] Osanna, P. H., Durakbasa, M.N., Kräuter, L., 2008, "Industrial Metrology and Interchangeable Manufacturing under the Viewpoint of Nanotechnology and Nanometrology", Bulgarian Academy of Sciences, Problems of Engineering Cybernetics and Robotics, Vol. 59, pp.60-73

[2] D. Whitehouse, "Comparison between stylus and optical methods for measuring surfaces",
Annals CIRP, 37(2), pp. 649-653, 1988

[3] ISO 4287 Geometrical Product Specifications (GPS) - Surface texture: Profile method-Terms, definitions and surface texture parameters (ISO 4287:1997+Cor 1:1998+Cor 2:2005+Amd 1:2009)

[4] EN ISO 1101-2006: Geometrical Product Specifications (GPS) - Geometrical Tolerancing - Tolerances of Form, Orientation, Location and Run-out; EN ISO 1101/A1: 2010 Geometrical Product Specifications (GPS) - Geometrical tolerancing-Tolerances of form, orientation, location and run-out- Amendment 1: Representation of specifications in the form of a $3 D$ model

[5] ISO 8015 - 2011: Geometrical product specifications (GPS) - Fundamentals - Concepts, principles and rules

[6] EN/ISO 9001 - 2008: Quality Management Systems - Requirements. 2008-12

[7] EN/ISO 14001 - 2009: Environmental management systems - Requirements with guidance for use (ISO 14001:2004 + Cor. 1:2009)

[8] EN/ISO 9004 - 2009: Managing for the sustained success of an organization - A quality management approach

[9] ISO/TR 14638: Geometrical product specification (GPS) - Masterplan

[10] ISO/TR 10360-1-1993, Part 1: Coordinate Metrology - Part 1: Definitions. Applications of the Fundamental Geometrical Principals. 1993

[11] P.H. Osanna, N.M. Durakbasa e.a.: Global Competitive Manufacturing on the Basis of Intelligent Metrology and Quality Management as Important Tools. Proceedings of the International Symposium "Tools and Methods of Competitive Engineering - TMCE'2002" (Editor: Horvath, I. e.a.), Wuhan, China, April 2002, ISBN 7-5609-2682-7, 837/844 
Acta Mechanica Slovaca

Journal published by Faculty of Mechanical Engineering - Technical University of Košice

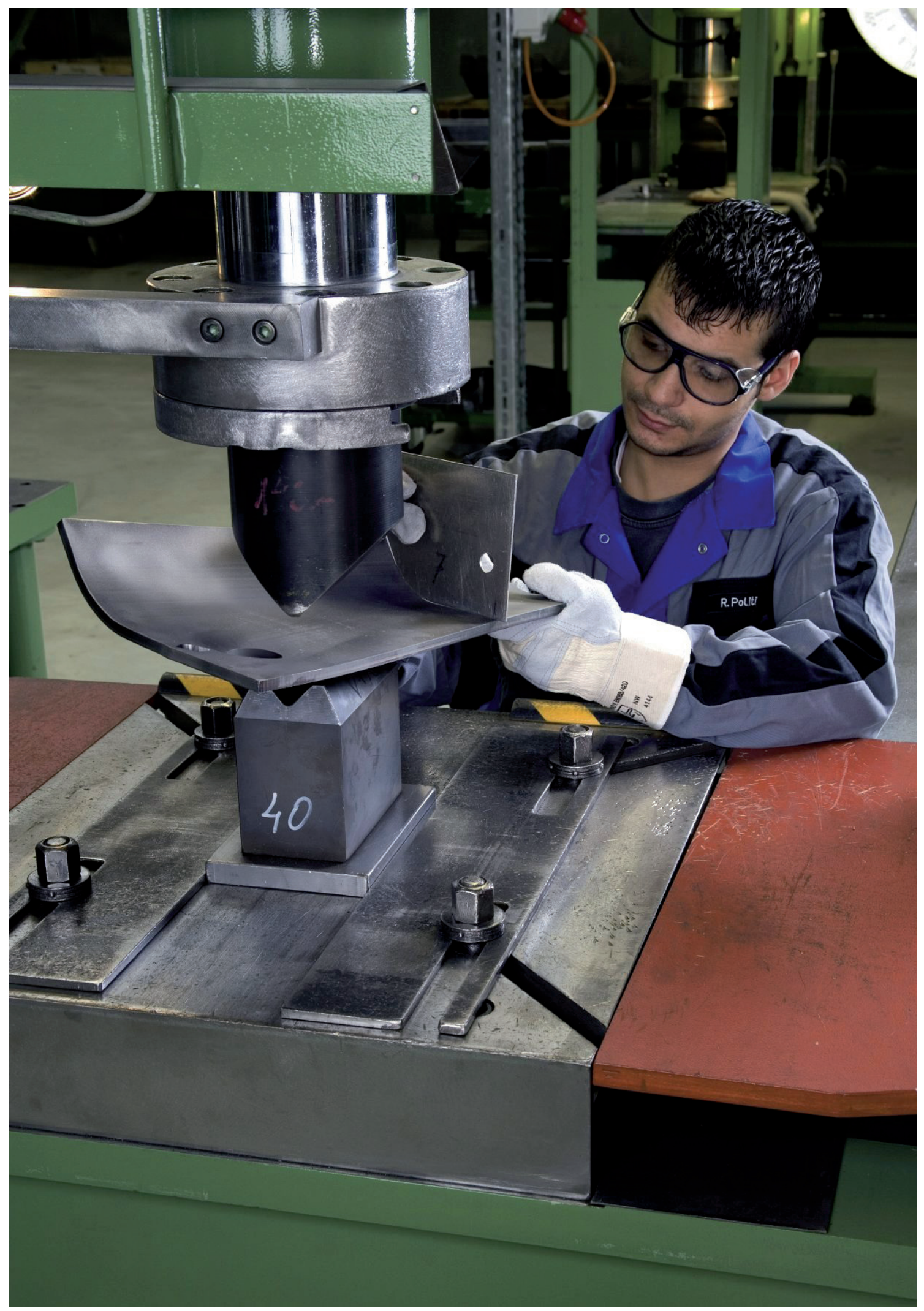

